\title{
X-ray scattering by interstellar dust grains as a diagnostic tool ${ }^{\star}$
}

\author{
J. Trümper
}

\author{
Max-Planck-Institut für Extraterrestrische Physik, Giessenbachstrasse, 85748 Garching, Germany \\ e-mail: jtrumper@mpe.mpg.de
}

Dust exists almost everywhere in the universe, except in its very early hot phase. It is produced by cool stars and constitutes an important component of the interstellar medium, and some of the dust even gets into intergalactic space. Dust also plays an extremely important role in the star and planet formation taking place in the huge dusty molecular clouds. The surface of dust grains is the place where some key chemical reactions in space occur, and in the end we ourselves are made of stardust.

To many astronomers dust is a nuisance since it obscures some of the more interesting places in the universe, such as the center of our Galaxy or the sites of star formation. On the other hand, the properties of dust reflect the complex processes of cosmic evolution, which change and recycle matter. And last but not least, the reddening and absorption of light by dust is very useful for many diagnostic purposes, in particular determining the distances to cosmic objects.

There are many different ways to investigate cosmic dust. The paper by Peter Predehl and Jürgen Schmitt on "X-raying the interstellar medium: ROSAT observations of dust-scattering halos" combines X-ray measurements of interstellar absorption and of dust scattering halos with measurements of interstellar reddening, using a sample of bright X-ray sources. The correlations between these three quantities are extremely useful for many astrophysicists dealing with problems of source distances or spectra, as well as with the physics of the interstellar medium. This is so obvious, while being underpinned by the large number of citations, that I will refrain from discussing the subject in any more detail. Instead I sketch the prehistory of this work and the scientific environment provided for it at the Max-Planck-Institut für Extraterrestrische Physik (MPE).

Like most scientists in the early days of high-energy astrophysics, we had been working in nuclear and cosmic ray physics before we became interested in X-ray astronomy in the late 1960s. After starting a balloon program in hard X-ray astronomy at the University of Tübingen, we began developing imaging X-ray telescopes in 1972, and in 1975 we proposed a satellite project, which finally became ROSAT. Already in $1972 / 73$, we were thinking about the use of such telescopes. Volker Schönfelder and I had discussions about observations of halos produced by small-angle scattering of X-rays by interstellar dust grains. We noted that in the case of variable sources (and most X-ray sources known at that time were

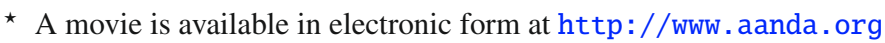

highly variable) the source variations would be damped out with increasing off-source angle. We proposed using this effect to measure the distances of X-ray sources (Trümper \& Schönfelder 1973). Heinrich Spiegelhauer, a Ph.D. student of mine, and I generalized the method by showing that one can derive not only source distances, but also the distribution of dust along the line of sight and even the grain size distribution (Spiegelhauer \& Trümper 1975), provided data were available with a high enough signal-to-noise ratio for such an analysis. As a member of the EXOSAT mission definition group, I had discussed using lunar and earth limb occultations to study the angular distribution of $\mathrm{X}$-ray halos. Lunar occultations had been a prime mission objective in the planning phase of this satellite, but when EXOSAT was in orbit, lunar pointings were actually avoided because of the potential danger of the bright moon for the star trackers. In collaboration with the late Hermann Debrunner, I owned a few accepted proposals on dust halos, which to our disappointment were never implemented.

In 1977 Peter Predehl joined our group at MPE as a Ph.D. student. The subject of his thesis dealt with the development of high-resolution grating spectrometers for a hypothetical successor to ROSAT, which was to survey X-ray spectra. Unfortunately this plan did not materialize, but these high-tech instruments were flown later as "Low Energy Transmission Gratings" on Chandra in collaboration with the Space Research Institute in Utrecht (Brinkman et al. 2000). Peter led the final calibration efforts at the long beam facility of the Marshall Space Flight Center, where the experience he had gained in ROSAT ground testing and other mission relevant activities turned out to be very useful.

I first met Jürgen Schmitt in 1983 at the Harvard Smithsonian Center for Astrophysics where he was working with Bob Rosner on a Ph.D. Thesis on the coronal emission of cool stars based on Einstein observations. I gave a talk about our ROSAT plans and discussed the first spectrally resolved pictures we had taken of Puppis A with a rocket-borne telescope. Jürgen was very excited about the prospects for ROSAT and he joined our group in 1984. His main duty in the project was to prepare the Announcements of Opportunity for the international guest-observer community and to develop the software for setting up an efficient observation timeline, which would minimize the slew times and maximize the time on targets. The resulting program turned out to be very efficient. A professor of mathematics offered to test his elaborated optimization program by setting up the 
ROSAT timeline. It turned out that it did not produce any higher efficiency than Jürgen's software.

On 1 June 1990 ROSAT was launched from Cape Canaveral. The launch was the main contribution by NASA, together with the HRI provided by Harvard-SAO. Two weeks later, on 16 June we had our first ROSAT light with the supernova 1987A as target, and everything worked fine. These were very happy times for all of us after many years of instrument and software development, testing, and scientific preparations in the dry-run mode.

In July 1990 Jürgen put a lunar pointing into the satellite's timeline as an attitude management and control system test. His method was very simple. He just switched off the star trackers for the duration of the observation. This maneuver resulted in a stunning first X-ray image of the Moon, which made it onto the title page of Nature (Schmitt et al. 1991). The beautiful picture showed the Sunlit lunar crescent and the shadow of the dark Moon cast onto the soft X-ray background. It was the first X-ray shadow ever seen in X-ray astronomy, and many X-ray shadow experiments followed showing the dark clouds of the cool interstellar medium along the Galactic equator. All this was made possible by the very low particle background of the ROSAT PSPC, which was about a factor of 10 below that of the Einstein IPC. To our surprise, the X-ray signal detected from the dark side of the Moon was much stronger than expected. But only a couple of years later, after the discovery of X-ray emission from comets by ROSAT in 1996, it became clear that these X-rays were originating between Moon and Earth - as an X-ray foreground - produced by the same mechanism as the X-rays from the comae of comets, namely by charge exchange between the ionized atoms of the solar wind with water vapor (comet) and the neutral gas corona (Earth). Another consequence of the successful lunar pointing was that Jürgen and Peter realized ROSAT's potential for direct imaging of dust-scattering halos.

A few months later the opportunity arose when the bright X-ray source (GX5-1) was occulted by the Moon and was caught in the act by ROSAT. The X-ray emission from the halo of GX5-1 was still visible despite GX5-1 having disappeared behind the lunar limb, and this allowed Peter and Jürgen to derive the radial distribution of the dust halo (Predehl et al. 1992). The excellent collaboration of Jürgen, Peter, and others in this experiment was the basis for their systematic study of dust halos with ROSAT, which is the main subject of this comment. Pioneering observations of dust halos had already been performed by Paul Gorenstein and Christopher Mauche, who studied six sources with the Einstein Observatory (Mauche et al. 1986). However, ROSAT with its PSPC offered a much better performance than Einstein with the IPC: at soft X-rays its collecting power was higher by a factor of 5, its spectral resolution by a factor of 4 , thus allowing precise measurements of the interstellar hydrogen column $N_{\mathrm{H}}$. And as already mentioned, the non X-ray background was about a factor 10 lower (per $\operatorname{arcmin}^{2}$ ). Another important advantage of ROSAT was that the scattering of X-rays by the mirror's micro-roughness was lower by a factor of 50 . And finally it was important that the dust halo and the interstellar absorption were measured simultaneously with the same instrument. In their ROSAT study of dust-scattering halos, Peter and Jürgen also came to a series of interesting conclusions on the physical nature of dust; e.g., they could prove the "fluffiness" of dust grains. As a neat side effect, they could also possibly explain why the explosion of the supernova in Cassiopeia (Cas A) had not been seen around 1680.

Near the end of the ROSAT mission, a unique opportunity came up to observe a lunar occultation of Sco X-1, which is possible only every sixteen years. Sco X-1 was the only X-ray source in the sky at which ROSAT had never been pointed, because the high flux could damage the PSPC. This complicated the planning of the observation that Peter Predehl was part of. As soon as Sco X-1 entered the PSPC field of view, the high voltage had to be switched off and turned on again when the bright source was a few arcsec behind the lunar rim. The maneuvre went off splendidly and resulted in a beautiful movie - one of the first X-ray movies in history ${ }^{1}$.

A new era of dust research began with the launches of Chandra and XMM-Newton. The old dream of Volker Schönfelder and myself of measuring distances of variable X-ray sources became true for the first time, when Peter analyzed Chandra data of Cyg X-3 and succeeded in determining the geometrical distance of this source. The reasons that the world had to wait 27 years (1973-2000) for such an experiment were the tight combination of source distance, the period of its brightness variability, and the angular resolution of the X-ray telescope. Although the radiation from Cyg X-3 had to travel around 25000 years, the scattered and therefore delayed radiation arrived only 15 min later and was observed within a circle of only 10 arcsec around the point source (Predehl et al. 2000).

A few years later, Peter and his Ph.D. student, Elisa Costantini, analyzed data on Cyg X-2 both with Chandra and XMM-Newton. For the first time, the spectrum of a scattered halo could be studied in detail, resulting in the detection of the signatures of the interstellar dust elements oxygen, magnesium, and silicon in the halo spectrum. Contributions of various silicates, e.g. olivines and pyroxenes, could be identified as well (Costantini et al. 2005). This was the first step by X-ray astronomy into the real chemistry of interstellar dust.

\section{References}

Trümper, J., \& Schönfelder, V. 1973, A\&A, 25, 445

Spiegelhauer, H., \& Trümper, J. 1975, JBIS, 28, 219

Brinkman, A. C., Gunsing, C. J. T., Kaastra, J. S., et al. 2000, ApJ, 530,2, 111

Schmitt, J. H. M. M., Snowden, S. L., Aschenbach, B., et al. 1991, Nature, 349, 583

Predehl, P., \& Schmitt, J. H. M. M. 1995, A\&A, 293, 889

Predehl, P., Schmitt, J. H. M. M., Snowden, S. L., \& Trümper, J. 1992, Science, 257, 935

Mauche, C. W., \& Gorenstein, P. 1986, ApJ, 302, 371

Predehl, P., Burwitz, V., Paerels, F., \& Trümper, J. 2000, A\&A, 357, L25

Costantini, E., Freyberg, M. J., \& Predehl, P. 2005, A\&A, 444, 187 